\title{
Theoretical study of electronic relaxation processes in hydrated $\mathrm{Gd}^{3+}$ complexes in solutions
}

\author{
S. Rast ${ }^{1, *}$, P.H. Fries ${ }^{1}$ and E. Belorizky ${ }^{2}$ \\ ${ }^{1}$ Laboratoire Reconnaissance lonique, Service de Chimie Inorganique et Biologique, \\ Département de Recherche Fondamentale sur la Matière Condensée, CEA-Grenoble, \\ 38054 Grenoble cedex 9, France \\ ${ }^{2}$ Laboratoire de Spectrométrie Physique, Université Joseph Fourier, \\ BP. 87, 38402 Saint-Martin-d'Hères cedex, France
}

\footnotetext{
* Correspondence and reprints.
}

Résumé : On réinterprète les largeurs de raie RPE des ions $\left[\mathrm{Gd}\left(\mathrm{H}_{2} \mathrm{O}\right)_{8}\right]^{3+}$ mesurées dans l'eau à différents champs magnétiques par le groupe de Merbach. On propose un nouveau modèle théorique de la relaxation électronique transverse. On montre que tous les termes statiques du champ cristallin, compatibles avec la symétrie du complexe, jouent un rôle non négligeable. On étudie aussi l'influence des fluctuations rapides de distorsion du complexe.

mots-clés: champ cristallin, théorie de la relaxation selon Redfield, largeur de raie.

Abstract: The EPR line widths of $\left[\mathrm{Gd}\left(\mathrm{H}_{2} \mathrm{O}\right)_{8}\right]^{3+}$ measured in water at various magnetic fields by Merbach's group have been reinterpreted. A theoretical model of the transverse electronic relaxation is proposed. All the terms of the static zero-field splitting (ZFS) allowed by the symmetry of the complex are included and shown to have a significant contribution. The influence of a transient distortion ZFS is also studied.

Key words: zero-field splitting, Redfield's relaxation theory, EPR line widths.

\section{INTRODUCTION}

Recently the investigation of paramagnetic molecules containing trivalent lanthanide aqua ions in solutions has been the object of a renewed interest. The whole lanthanide series has been studied. The various studies include NMR relaxation of 
water solvent $[1,2]$ and of probe solutes $[3,4,5]$ in $\mathrm{D}_{2} \mathrm{O}$ solutions, in addition to EPR studies of gadolinium (III) complexes [6]. The main problem arises from the electronic relaxation of these systems, the mechanisms of which are not yet fully understood and need further experimental and theoretical studies. The aim of this note is to sketch a general theoretical formalism for treating the electronic relaxation of paramagnetic ion complexes within the Redfield approximation, and to present a new interpretation of the very detailed measurements of EPR line widths of $\mathrm{Gd}^{3+}$ aqua ions carried out by Powell et al. [6] at various temperatures and magnetic fields. In their paper, these authors interpreted the experimental line widths by using an ad hoc parameterized formula which accounts for the magnitude $\Delta$ of a simple zerofield splitting (ZFS) acting on the $\mathrm{Gd}^{3+}$ spin $\mathrm{S}=7 / 2$ and for the unique correlation time $\tau_{v}$ that characterizes the fluctuations of this ligand field. They assumed an Arrhenius temperature dependence for $\tau_{v}$. Here, in the reference frame of the aqua complex, we use the most general form of the static ligand field Hamiltonian $\mathcal{H}_{1}$ that is compatible with the symmetry of the complex. Then, in the laboratory frame, we solve the Redfield equations for the EPR relaxation by assuming that $\mathcal{H}_{1}(t)$ fluctuates because of the random reorientation of the complex given in a first approximation by the solutions of the usual isotropic rotational diffusion equation. Such a picture is supported by the molecular dynamics (MD) calculations of Kowall et al. $[7,8]$ who showed that the eight coordinating water molecules around $\mathrm{Gd}^{3+}$ are located on average at the corners of a square antiprism and that this structure undergoes random pseudorotations, transient distortions, and exchanges of water molecules.

\section{THEORY}

Let $\omega_{S}=2 \mu_{B} H$ be the Larmor frequency of the $\mathrm{Gd}^{3+}$ spin. The Hamiltonian is

$$
\hbar \mathcal{H}=\hbar \omega_{S} S_{z}+\hbar \mathcal{H}(t),
$$


where $\hbar \omega_{S} S_{Z}$ is the Zeeman term which is quantitized in the laboratory frame, and $\mathcal{H}_{1}(t)$ is the static averaged ligand field perturbation which is quantitized in the rotating molecular frame $X Y Z$. In the latter, according to the square antiprism symmetry, we have

$$
\mathcal{H}_{1}=B_{2} T_{0}^{2}+B_{4} T_{0}^{4}+B_{6} T_{0}^{6} .
$$

The $T_{q}^{k}$ are linear combinations of products of the components $S_{z}, S_{ \pm}$of the $\mathrm{Gd}^{3+}$ $S=7 / 2$ spin in the molecular frame, transforming as the standard components of an irreducible tensor of order $k[9,10]$. For the $f$ electrons of the studied ground multiplet ${ }^{8} S_{7 / 2}$, the real coefficients $B_{2}, B_{4}, B_{6}$ define the magnitudes of the second, fourth, and sixth order terms of the ligand field which indirectly stems from the ligand field acting on the excited multiplets through the spin orbit coupling [11]. The precise computation of these coefficients is a very difficult task so that they are considered as adjustable parameters. The terms $T_{q}^{k}$ with $q= \pm 4$ do not appear in expression (2) because the molecular coordinate system $X Y Z$ was chosen so that its $Z$-axis is the $C_{4}$-symmetry axis and its $X$ - and $Y$-axes are mutually orthogonal $C_{2}$ axes.

Because the orientational correlation times $\tau_{k}^{-1}=k(k+1) D^{r}$ are such that $\left\|H_{1}(t) \tau_{k}\right\|<1$, as $\left\|H_{1}\right\| \approx 2 \cdot 10^{10} \mathrm{rad} / \mathrm{s}$ and $\tau_{k}<10^{-11} \mathrm{~s}$, we use the Redfield formalism for studying the electronic spin relaxation.

Expressing $\mathcal{H}_{1}(t)$ in the laboratory frame we get 


$$
\mathcal{H}_{1}(t)=\sum_{k=2,4,6} \sum_{q=-k}^{k} B_{k} T_{q}^{k} R_{q 0}^{k}\left(\Omega_{t}\right)
$$

where the $T_{q}^{k}$ are irreducible tensor operators of the spin in the laboratory frame and $R_{q 0}^{k}\left(\Omega_{t}\right)$ are elements of the Wigner matrices of the random rotation $\Omega_{t}$ transforming the laboratory frame into the molecular frame at time $t$.

In the high temperature limit, the EPR absorption curve is given by the Fourier transform of the relaxation function $[12,6]$

$$
\mathrm{G}(t)=\frac{1}{2 S+1} \operatorname{tr}\left(S_{+}(t) S_{-}(0)\right)
$$

where $S_{+}(t)=\exp \left(i \omega_{S} S_{z} t\right) S_{+}^{*}(t) \exp \left(-i \omega_{S} S_{z} t\right)$, and $S_{+}^{*}(t)$ obeys the differential equation

$$
\frac{1}{i} \frac{d S_{+}^{*}}{d t}=\left[\exp \left(-i \omega_{S} S_{z} t\right) \mathcal{H}_{1}(t) \exp \left(i \omega_{S} S_{z} t\right), S_{+}^{*}(t)\right]
$$

We are led to a master equation

$$
\frac{d\left(S_{+}^{*}\right)_{M M^{\prime}}}{d t}=\sum_{M_{1} M_{1}^{\prime}} R_{M M^{\prime}, M_{1} M_{1}^{\prime}}\left(S_{+}^{*}\right)_{M_{1} M_{1}^{\prime}}
$$

where the magnetic quantum numbers verify $-7 / 2 \leq M, M^{\prime}, M_{1}, M_{1}{ }^{\prime} \leq 7 / 2$, and the sum is limited according to the condition $E_{M_{1}}-E_{M_{1}^{\prime}}=E_{M}-E_{M^{\prime}}=\hbar \omega_{S}$ for the Zeeman energies. 
Assuming an isotropic rotational diffusion of the complex, the Redfield matrix elements $R_{M M^{\prime}, M_{1} M_{1}}$ can be expressed as linear combinations of spectral densities $\mathrm{J}^{(k)}\left(n \omega_{S}\right)(n$ integer $\leq 6)$, which are the Fourier transforms of the time correlation functions of the Wigner matrix elements

$$
\left\langle\overline{R_{q 0}^{k}\left(\Omega_{t}\right)} R_{q^{\prime} 0}^{k^{\prime}}\left(\Omega_{t=0}\right)\right\rangle=\frac{1}{2 k+1} \exp \left(-t / \tau_{k}\right) \delta_{k k^{\prime}} \delta_{q q^{\prime}}
$$

Thus, $\mathrm{G}(t)$ and the EPR absorption line can be written in terms of $\left|B_{2}\right|^{2},\left|B_{4}\right|^{2},\left|B_{6}\right|^{2}$ and of the three correlation times $\tau_{2}, \tau_{4}=3 \tau_{2} / 10, \quad \tau_{6}=\tau_{2} / 7$ with $\tau_{2}=\tau_{2}^{298} \exp \left[\frac{E_{A}}{R}\left(\frac{1}{T}-\frac{1}{298.15 \mathrm{~K}}\right)\right], \tau_{2}^{298}$ being the value of $\tau_{2}$ at $298.15 \mathrm{~K}$ and $E_{A}$ the activation energy for the rotation of the complex.

\section{RESULTS AND DISCUSSION}

In figure la we display the peak-to-peak EPR line widths for $\left[\mathrm{Gd}\left(\mathrm{H}_{2} \mathrm{O}\right)_{8}\right]^{3+}$ measured by Powell et al. [6] versus the inverse of temperature for magnetic fields ranging between 0.14 and 5.0 Tesla in the low concentration limit, together with the calculated values obtained with the above model by fitting $\left|B_{2}\right|^{2},\left|B_{4}\right|^{2},\left|B_{6}\right|^{2}, \tau_{2}^{298}$, and $E_{A}$. The best agreement was obtained with $\left|B_{2}\right|=0.72 \cdot 10^{10} \mathrm{rad} / \mathrm{s}$, $\left|B_{4}\right|=0.049 \cdot 10^{10} \mathrm{rad} / \mathrm{s}, \quad\left|B_{6}\right|=0.026 \cdot 10^{10} \mathrm{rad} / \mathrm{s}, \quad \tau_{2}^{298}=6.4 \cdot 10^{-12} \mathrm{~s}, \quad$ and $E_{A}=13.5 \mathrm{~kJ} / \mathrm{mol}$.

A reasonable agreement is reached with however some discrepancy at high fields. Interestingly, although our model cannot provide the signs of $B_{2}, B_{4}, B_{6}$, we obtain total crystal field splittings $\Delta_{\text {tot }}\left(B_{2}, B_{4}, B_{6}\right)$ of the ground ${ }^{8} \dot{S}_{7 / 2}$ multiplet ranging between 0.7 and $0.8 \mathrm{~cm}^{-1}$ depending on the relative signs chosen before $\left|B_{2}\right|,\left|B_{4}\right|$, 


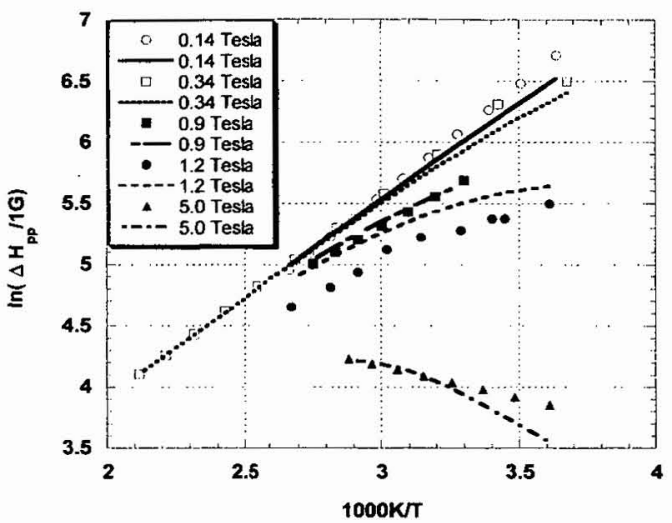

Figure 1a.

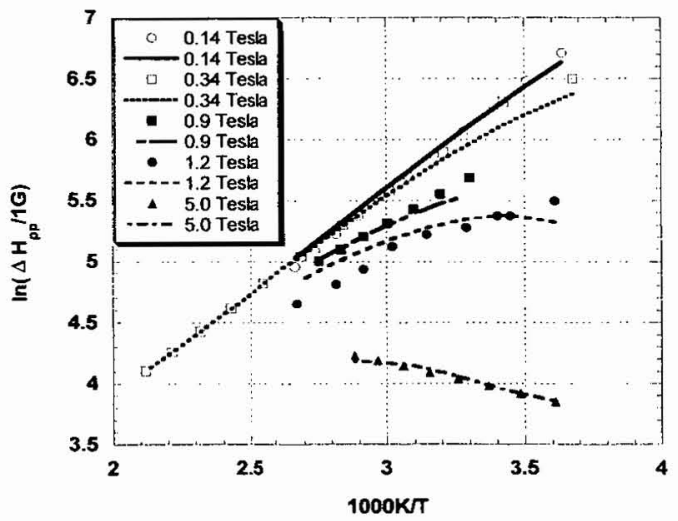

Figurelb. Logarithm of the peak-to-peak line width versus inverse temperature. Symbols: experimental values. Lines: Theory. Without (a) and with (b) transient ZFS contribution. 
$\left|B_{6}\right|$. This range of values is quite reasonable and comparable to the ZFS observed for $\mathrm{Gd}^{3+}$ ions in solids. It should be noted that with the above values of $\left|B_{2}\right|,\left|B_{4}\right|,\left|B_{6}\right|$ the respective contributions of the second, fourth, and sixth order crystal field parameters to $\Delta_{\text {tot }} \quad$ are $\quad \Delta_{\text {tot }}\left(\left|B_{2}\right|, 0,0\right)=0.56 \mathrm{~cm}^{-1}, \quad \Delta_{\text {tot }}\left(0,\left|B_{4}\right|, 0\right)=0.20 \mathrm{~cm}^{-1}, \quad$ and $\Delta_{\text {tot }}\left(0,0,\left|B_{6}\right|\right)=0.40 \mathrm{~cm}^{-1}$, showing that the fourth and sixth order terms of the ligand field cannot be neglected.

In order to improve the fit in the high field region we introduced another relaxation mechanism currently invoked for these hydrated complexes, i. e. the transient fluctuations of the ligand field due to the collisions of the surrounding water molecules and somewhat to the exchange of the coordinating water molecules $[7,8]$. This mechanism is rather complex. Following a standard procedure, in order to avoid an excess of unknown parameters, it was simply accounted for by introducing an additional stochastically independent term in $\mathcal{H}_{1}(t)$

$$
V(t)=D(t)\left[S_{z}^{2}-S(S+1) / 3\right]+E(t)\left[S_{x}^{2}-S_{y}^{2}\right]
$$

with $D(t)$ and $E(t)$ jumping in a random way between two sets of values $\pm(D, E)$ with a correlation time

$$
\tau_{v}=\tau_{v}^{298} \exp \left[\frac{E_{v}}{R}\left(\frac{1}{T}-\frac{1}{298.15 \mathrm{~K}}\right)\right]
$$

As shown in figure $1 \mathrm{~b}$, the EPR experimental line widths are well reproduced by the new set of parameters $\left|B_{2}\right|=0.27 \cdot 10^{10} \mathrm{rad} / \mathrm{s}, \quad\left|B_{4}\right|=0.053 \cdot 10^{10} \mathrm{rad} / \mathrm{s}$, $\left|B_{6}\right|=0.023 \cdot 10^{10} \mathrm{rad} / \mathrm{s}, \quad \tau_{2}^{298}=1.6 \cdot 10^{-11} \mathrm{~s}, \quad E_{A}=15.2 \mathrm{~kJ} / \mathrm{mol}$, $\Delta=\sqrt{2 D^{2} / 3+E^{2}}=0.47 \cdot 10^{10} \mathrm{rad} / \mathrm{s}, \tau_{v}^{298}=3.1 \cdot 10^{-14} \mathrm{~s}, E_{v}=1.0 \mathrm{~kJ} / \mathrm{mol}$. 
The values of the ZFS parameters remain comparable to those previously determined and the correlation time $\tau_{v}$ of the transient process appears to be much shorter than the rotational correlation time $\tau_{v}$.

\section{CONCLUSION}

We have underlined the importance of the fourth and sixth order terms of the ZFS on the electronic relaxation of the $\mathrm{Gd}^{3+}$ ion. The correlation time found for the Brownian rotation of the complex in $\mathrm{H}_{2} \mathrm{O}$ has a reasonable value. This study will be completed by a further analysis of the complete line shape and by a detailed study of the NMR relaxation dispersion of nuclei located in suitable probe solutes $[3,5]$.

\section{REFERENCES}

[1] Bertini I., Capozzi F., Luchinat C., Nicastro G., Xia Z. (1993) J. Phys. Chem. 97 24, 6351-6354.

[2] Powell D.H., Dhubhgaill O.M.N., Pubanz D., Helm L., Lebedev Y.S., Schlaepfer W., Merbach A.E. (1996) J. Am. Chem. Soc. 118, 39, 9333-9346.

[3] Vigouroux C., Bardet M., Belorizky E., Fries P.H., Guillermo A., (1998) Chem. Phys. Lett. 286, 93-100.

[4] Vigouroux C., Belorizky E., Fries P.H. (1999) Eur. Phys. J. D 5, 243-255.

[5] Dinesen T.R.J., Bryant R.G., (1999) Chem. Phys. Lett. (in press).

[6] Powell D.H., Merbach A.E., González G., Brücher E., Mickskei K., Ottaviani M.F., Köhler K., von Zelewsky A., Grinberg O.Y., Lebedev Y.S., (1993) Helv. Chim. Acta 76 2129-2146.

[7] Kowall Th., Foglia F., Helm L., Merbach A.E., (1995) J. Phys. Chem. 99, 35, 13078-13087.

[8] Kowall Th., Foglia F., Helm L., Merbach A.E., (1995) J. Am. Chem. Soc. 117, 13, 3790-3799.

[9] Buckmaster H.A., Chatterjee R., Shing Y.H., (1972) Phys. Stat. Sol. (a) 13, 9-49. [10] Messiah A., (1972) Mécanique Quantique. Dunod, Paris, Tome II, 918-926 p.

[11] Abragam A., Bleaney B. (1971) Résonance Paramagnétique Electronique des Ions de Transition. Presses Universitaires de France, Paris, 335-340 p.

[12] Abragam A., (1961) Les Principes du Magnétisme Nucléaire. Presses Universitaires de France, Paris, 440-441 p. 